\title{
Is Vertebral Artery Hypoplasia a Predisposing Factor for Posterior Circulation Cerebral Ischemic Events? A Comprehensive Review
}

\author{
Aristeidis H. Katsanos ${ }^{a}$ Maria Kosmidou $^{b}$ Athanassios P. Kyritsis $^{a}$ \\ Sotirios Giannopoulos ${ }^{a}$ \\ ${ }^{a}$ Department of Neurology and ${ }^{b} 1$ st Division of Internal Medicine, University of loannina School of Medicine, \\ loannina, Greece
}

\section{Key Words}

Vertebral artery hypoplasia - Ischemic stroke $\cdot$ Transient ischemic attacks · Posterior circulation stroke $\cdot$ Brainstem infarction $\cdot$ Cerebellar infarction $\cdot$ Cerebral blood flow

\begin{abstract}
Vertebral artery hypoplasia is not currently considered an independent risk factor for stroke. Emerging evidence suggest that vertebral artery hypoplasia may contribute to posterior circulation ischemic events, especially when other risk factors coexist. In the present literature review, we present published data to discuss the relationship between a hypoplastic vertebral artery and posterior circulation cerebral ischemia. Despite difficulties and controversies in the accurate definition and prevalence estimation of vertebral artery hypoplasia, ultrasound studies reveal that the reduced blood flow observed ipsilateral to the hypoplastic vertebral artery may result in local cerebral hypoperfusion and subsequent focal neurological symptomatology. That risk of cerebral ischemia is related to the severity of the hypoplasia, suggesting that the smaller of paired arteries are more vulnerable to occlusion. Existing cohort studies further support clinical observations that hypoplastic vertebral artery enhances synergistically the vascular risk for posterior circulation ischemic events and is closely associated with both atherosclerotic and prothrombotic processes.

Copyright $\odot 2013$ S. Karger AG, Basel
\end{abstract}

\section{Introduction}

Congenital anatomical variations of both vertebral arteries are relatively frequent; left vertebral artery dominance presents in $50 \%$ of the population, while similar size vertebral arteries present with an only $25 \%$ prevalence. Due to this high prevalence, vertebral artery hypoplasia (VAH) has not yet been considered an independent risk factor for ischemic stroke $[1,2]$. However, emerging evidence from case reports (table 1) and recent imaging (table 2) and cohort studies (table 3 ) suggest that VAH may contribute to ischemic events, even in young patients, especially when other risks factors are present [3].

In the present literature review, we present published data from case reports, imaging protocols and cohort studies to discuss thoroughly the relationship between $\mathrm{VAH}$ and posterior circulation cerebral ischemia. Our literature search through Medline and Embase was based on the combination of terms: vertebral artery hypoplasia, stroke, transient ischemic attacks, brainstem and cerebellar infarcts, and posterior circulation ischemia. References of retrieved articles were also screened. Only papers in English were included. The last search was done in PubMed on October 13, 2012.

\section{KARGER}

E-Mail karger@karger.com

www.karger.com/ene (c) 2013 S. Karger AG, Basel

0014-3022/13/0702-0078\$38.00/0
Aristeidis H. Katsanos, MD

Department of Neurology

University of Ioannina, School of Medicine

University Campus, GR-45110 Ioannina (Greece)

E-Mail ar.katsanos@gmail.com 
Table 1. Case reports from patients with vertebral artery hypoplasia and posterior cerebral ischemia

\begin{tabular}{lcllll}
\hline Author & Age & Sex & VAH & Risk factors & Imaging findings \\
\hline Giannopoulos et al. [3] & 37 & M & unilateral & hypertension, dyslipidemia & lateral medular infarction \\
\hline Giannopoulos et al. [3] & 38 & M & unilateral & hypertension, Crohn's disease & lateral medular infarction \\
\hline Giannopoulos et al. [3] & 40 & F & unilateral & hypertension & lateral medular infarction \\
\hline Kawakami et al. [8] & 8 & M & unilateral & sport injury & cerebellar infarct \\
\hline Mestan [7] & 67 & F & bilateral & - & cerebellar infarct \\
\hline Orimo et al. [5] & 36 & M & bilateral & hypertension, hypercholesterolemia & $\begin{array}{l}\text { pons, medulla oblongata and } \\
\text { right cerebellar infarctions }\end{array}$ \\
\hline Tai et al. [6] & 60 & $\mathrm{M}$ & bilateral & $\begin{array}{l}\text { hypertension, hypercholesterolemia, } \\
\text { history of TIA }\end{array}$ & bilateral medial medulla infarcts \\
\hline
\end{tabular}

Table 2. Imaging findings from ultrasound protocols in patients with vertebral artery hypoplasia

\begin{tabular}{|c|c|c|c|c|c|c|}
\hline Author & $\begin{array}{l}\text { Patients } \\
\text { no }\end{array}$ & $\begin{array}{l}\text { Patients } \\
\text { status }\end{array}$ & $\begin{array}{l}\text { Imaging } \\
\text { method }\end{array}$ & VAH definition & VAH percentage & Main outcome \\
\hline Chen et al. [12] & 1,000 & $\begin{array}{l}\text { free } \\
\text { of CVD }\end{array}$ & TCD & $<2.5 \mathrm{~mm}$ & $\begin{array}{l}9.2 \% \\
(5.9 \% \mathrm{R}-3.3 \% \mathrm{~L})\end{array}$ & $\begin{array}{l}\text { patients with VAH had significantly } \\
\text { lower MnfV and higher frequency of VA } \\
\text { flow insufficiency }\end{array}$ \\
\hline Jeng et al. [27] & 447 & $\begin{array}{l}\text { free } \\
\text { of CVD }\end{array}$ & TCD & $<2.2 \mathrm{~mm}$ & $\begin{array}{l}11.6 \% \\
(7.8 \% \mathrm{R}-3.8 \% \mathrm{~L})\end{array}$ & $\begin{array}{l}\text { reduced MFV ispilateral to the VAH and } \\
\text { larger MFV contralateral to the VAH }\end{array}$ \\
\hline $\begin{array}{l}\text { Lovrencic-Huzjan } \\
\text { et al. [10] }\end{array}$ & 59 & migraine & TCD & - & $36 \%$ & $\begin{array}{l}\text { higher frequency of } \mathrm{VAH} \text { in migraine } \\
\text { with aura patients }\end{array}$ \\
\hline Min et al. [13] & 410 & $\begin{array}{l}\text { free } \\
\text { of CVD }\end{array}$ & $\begin{array}{l}\text { TCD, } \\
\text { MRA }\end{array}$ & $\begin{array}{l}<50 \% \text { of the } \\
\text { contralateral side }\end{array}$ & $\begin{array}{l}3.4 \% \\
(2.14 \% \mathrm{R}-1.26 \% \mathrm{~L})\end{array}$ & $\begin{array}{l}\text { increased MFV of unilateral VA may } \\
\text { indicate contralateral VAH or VAP }\end{array}$ \\
\hline Wang et al. [9] & 82 & $\begin{array}{l}\text { children } \\
\text { with PCI }\end{array}$ & TCD & - & $7.3 \%$ & $\begin{array}{l}\text { decreased VA diameter related with } \\
\text { reduced blood flow and inadequate } \\
\text { blood supply to the posterior circulation }\end{array}$ \\
\hline
\end{tabular}

\section{Case Reports' Hypothesis: Vertebral Artery Hypoplasia May Lead to Posterior Circulation Ischemia, when Combined with Conventional Risk Factors}

Giannopoulos et al. [3] have reported three cases of young adults (average age 38 years) with lateral medullar ischemic events and associated these events with the presence of an ipsilateral hypoplasic vertebral artery on MRA. All 3 patients had two additional atherosclerotic or non- atherosclerotic risk factors for stroke, suggesting that vertebral artery hypoplasia combined with other conventional risk factors may provide an optimal background for brainstem ischemia. None of these patients had recurrent transient ischemic attack (TIA) or stroke after the secondary stroke prevention treatment and none of them had on MRI scans other ischemic strokes in other vascular territories, except those in the lateral medulla. The authors concluded that both their uneventful outcome and medical history further support their initial hypothesis 
Table 3. Cohort Studies from patients with vertebral artery disease and cerebral ischemia

\begin{tabular}{llllll}
\hline Author & $\begin{array}{l}\text { Stroke } \\
\text { subtype }\end{array}$ & $\begin{array}{l}\text { Number } \\
\text { of patients }\end{array}$ & VA abnormality & Risk factors & Main outcome \\
\hline $\begin{array}{l}\text { Chuang } \\
\text { et al. [19] }\end{array}$ & IS & 191 & $\begin{array}{l}\text { VAH }(<3 \mathrm{~mm}) \\
11.51 \%\end{array}$ & $\begin{array}{l}53.4 \% \text { one and } 42.4 \% \\
\text { more than two risk factors }\end{array}$ & $\begin{array}{l}\text { VAH was significantly more } \\
\text { frequent in brainstem/cerebellar } \\
\text { infarction }\end{array}$ \\
\hline $\begin{array}{l}\text { Delcker } \\
\text { et al. [15] }\end{array}$ & $\begin{array}{l}\text { TIA } \\
\text { (VBS })\end{array}$ & 62 & VAH $(<2 \mathrm{~mm})$, & $\begin{array}{l}\text { carotid artery } \\
\text { atherosclerosis }\end{array}$ & $\begin{array}{l}\text { patients with combined carotid and } \\
\text { VA disease had increased TIAs }\end{array}$ \\
$\begin{array}{l}\text { Hu et al. } \\
\text { [20] }\end{array}$ & IS & 841 & VAH $(<2 \mathrm{~mm})$ & - & $\begin{array}{l}\text { VAH was an independent risk factor } \\
\text { for PCS }\end{array}$ \\
\hline $\begin{array}{l}\text { Lochner } \\
\text { et al. [24] }\end{array}$ & IS or & 88 & $\begin{array}{l}\text { VAH }(<2 \mathrm{~mm} \text { or }>50 \% \\
\text { TIAfference in VA })\end{array}$ & $\begin{array}{l}\text { FTC }(100 \%), \\
\text { atherosclerosis in VA, BA }\end{array}$ & $\begin{array}{l}\text { the simultaneous occurrence of VAH } \\
\text { and F may predispose to PCS }\end{array}$ \\
\hline $\begin{array}{l}\text { Park } \\
\text { et al. [17] }\end{array}$ & $\begin{array}{l}\text { PICAI } \\
\text { or LMI }\end{array}$ & 529 & $\begin{array}{l}\text { VAH }(<2 \mathrm{~mm}) 35.2 \% \\
(3.4 \% \text { bilaterally })\end{array}$ & - & $\begin{array}{l}\text { both PICAI and LMI were dominant } \\
\text { in patients with VAH }\end{array}$ \\
\hline $\begin{array}{l}\text { Perren } \\
\text { et al. [18] }\end{array}$ & IS & 725 & VAH $(<2.5 \mathrm{~mm})$ & $\begin{array}{l}\text { risk factors equally } \\
\text { distributed }\end{array}$ & $\begin{array}{l}\text { patients with VAH had significantly } \\
\text { more frequent PCS }\end{array}$ \\
\hline $\begin{array}{l}\text { Toghi } \\
\text { et al. [16] }\end{array}$ & CI & 293 & VAH, stenosis or & - & $\begin{array}{l}\text { 2/3 of the patients with SCA and } \\
\text { AICA infarcts had unilateral VA } \\
\text { occlusion or severe stenosis }\end{array}$ \\
\hline
\end{tabular}

VA = Vertebral artery; IS = ischemic stroke; VAH = vertebral artery hypoplasia; TIA = transient ischemic attack; VBS = vertebrobasilar system; FTC = fetal type circulation; BA = basilar artery; PCS = posterior circulation ischemia; $\mathrm{PICAI}=$ posterior inferior cerebellar artery infarction; LMI = lateral medulla infarction; $\mathrm{CI}=$ cerebellar infarction; SCA = superior cerebellar artery; AICA = anterior inferior cerebellar artery.

that their strokes can be attributed to the presence of a hypoplastic vertebral artery [4].

Locked-in syndrome due to brainstem infarction has been reported by Orimo et al. [5] in a 36-year-old male with hypertension and hypercholesterolemia. Brain MRI imaging showed pons, medulla oblongata and right cerebellum infraction, while cerebral angiography revealed bilateral VAH, a persistent right primitive trigeminal artery and retrograde blood flow of basilar artery from the primitive trigeminal artery.

Tai et al. [6] have recently presented a case of bilateral medial medullary infarction in a 60-year-old man with $\mathrm{VAH}$, uncontrolled hypertension, hypercholesterolemia and a single episode of transient ischemic attack 15 years ago. MRA confirmed the presence of a hypoplastic left vertebral artery with atherosclerotic changes, occlusion after the posterior inferior cerebral artery and stenosis of the proximal basilar artery. Bilateral hypoplasia - an uncommon vertebral artery anomaly with an estimated frequency of $0.75 \%$ - has also been revealed in a 67 -year-old female patient with cerebellar infarction by Mestan [7]. However, the angiogram in this particular case report failed to display any evidence of vertebral atherosclerotic occlusive disease.

Finally, Kawakami et al. [8] considered VAH to be a predisposing factor for ipsilateral vertebral artery dissection and concomitant cerebellar infarction after sport injury in an 8-year-old boy.

\section{Imaging Studies Illustrate that Neurological Symptoms in Subjects with Vertebral Artery Hypoplasia Are due to Reduced Blood Flow and Subsequent Cerebral Hypoperfusion}

Decreased vertebral artery diameter has been associated with both reduced blood flow and inadequate blood supply to the posterior circulation in a sonographic evaluation of pediatric patients with a history of headache, vertigo or syncope [9]. The role of VAH in the pathogenesis of migraine has also been examined with color Doppler ultrasound in 59 patients with migraine. Migraine with aura patients had a higher frequency of VAH (29\%) compared to migraine without aura patients (7\%), sug-
80

Eur Neurol 2013;70:78-83

DOI: $10.1159 / 000351786$
Katsanos/Kosmidou/Kyritsis/ Giannopoulos 
gesting that VAH presumably contributes to the hypoperfusion in the posterior circulation during the aura phase [10]. Even further, Simon et al. [11] have also proposed VAH as the main etiologic vascular factor for the marked ispilateral cerebellar hypoplasia that was observed in a 48-year-old patient.

In a retrospective analysis of color Doppler ultrasonography data, healthy subjects with VAH had significantly lower mean flow volume (MFV) in the hypoplastic vertebral artery and slightly increased MFV in the contralateral one, when compared to those without VAH. The mean net flow volume (MnFV) - the sum of the MFV of bilateral VA - was also significantly lower and the prevalence of vertebral artery flow volume insufficiency was, respectively, higher in subjects with unilateral VAH. Moreover, both the MFV and the MnFV were found to have a strong positive relation with the diameter of the vertebral artery [12]. Likewise, Min et al. [13] showed that the MFV was augmented in the contralateral (nonhypoplastic) side of the vertebral artery hypoplasia or aplasia, although the respective ipsilateral decrease in MFV in the hypoplastic artery did not reach statistical significance in their study.

Despite literature controversy, embolism and impaired vertebral blood flow volume are considered the major mechanisms of vertebrobasilar ischemia [14]. When blood flow on the one VA is temporarily reduced, the flow on the opposite VA is compensatory augmented to provide sufficient flow in the basilar artery. However, under certain prices in the diameter of the VAH the blood flow is reduced to a greater degree that results in unbalanced hemodynamics and in inadequate blood supply to the brain [9].

\section{Cohort Studies Further Test and Support the Hypothesis}

Patients with combined carotid and vertebral artery disease were found to have an increased incidence of TIAs in the vertebrobasilar system (71\%), when compared with patients with isolated carotid artery disease (8\%). This pronounced difference highlighted the dominant role of vertebral arteries in the pathogenesis of TIA in the posterior circulation [15]. Surprisingly, in another large multicenter study vertebral artery abnormalities were more strongly related with cerebellar ischemia when compared with the abnormalities of cerebellar arteries that distribute directly to the ischemic areas. More specifically, two thirds of the patients with superior cere-

VAH and Posterior Circulation Cerebral Ischemia bral artery or anterior inferior cerebral artery infarct had unilateral vertebral artery occlusion or severe stenosis [16].

A retrospective analysis of 529 patients with ischemic stroke by Park et al. [17] demonstrated that even though $\mathrm{VAH}$ is a common finding in the asymptomatic population, it is highly associated with posterior circulation strokes. Perren et al. [18] confirmed that among 725 firstever stroke patients, those with posterior circulation strokes had significantly more frequent VAH (13\%) compared to those with stroke in other territories (4.6\%). Similarly, Chuang et al. [19] reported that the overall incidence of unilateral VAH, measured in a study group of 191 acute ischemic stroke patients, was $11.5 \%$ and this percentage was significantly higher in cases of brainstem or cerebellar infarction. More than half of the study group patients had a single vascular risk factor and $79.4 \%$ of them had an additional vascular risk factor. Finally, a very recent retrospective study by $\mathrm{Hu}$ et al. [20] found a similar prevalence of VAH (10.8\%) in Chinese patients with acute ischemic stroke. The presence of VAH also appeared to be an independent risk factor for posterior cerebral ischemia, after multivariate logistic regression analysis.

Even in a pediatric population of children between 3 and 14 years old, hypoplasia of the vertebrobasilar system accounted for $3 \%$ of the total cerebrovascular hypoplasias, and VAH particularly was regarded as the main cause for transient ischemic attacks [21]. Additional reports suggested that children with congenital hypoplastic vertebral artery were more susceptible to arteriosclerosis either at a young age or later in adulthood $[9,14,22]$.

Park et al. [23] demonstrated in another study protocol that most of the 37 acute stroke patients with vertebral artery diameter of less than $3 \mathrm{~mm}$ in the V2 segment had relatively small or scattered lesions in the VA territory (cerebellum and/medulla), when compared with stroke patients without small vertebrobasilar system. The most common angiographic finding in patients with small vertebral artery and/or small basilar artery was VA stenosis or occlusion and the most common vascular risk factors were hypertension (83.3\%) and diabetes (22.2\%). All these patients had fetal posterior circulation, with an absent or hypoplastic P1 segment of the posterior cerebral artery, supporting the hypothesis that their vertebral arteries must be congenitally small rather than acquired. In the FTP circle of Willis there is an embryonic derivation of the posterior cerebral arteries from the internal carotid artery instead from the basilar artery, or alternatively there is a communication with the basilar artery through 
a hypoplastic P1 segment of the PCA. The presence of the aforementioned nonfunctional collateral pathway was strongly associated with both a hypoplastic vertebrobasilar system and posterior circulation ischemia in another case series of 13 patients [24].

Both studies by Park et al. [17, 23] and Chuang et al. [19] converge that the majority of patients with VAH related ischemic stroke is based on large-artery atherosclerosis. A hypoplastic vertebral artery, because of its decreased flow volume and flow velocities, could presumably be more susceptible to prothrombotic or atherosclerotic processes than normal vertebral arteries. Blood rheology at a low shear rate was found significantly impaired in symptomatic patients with VAH compared to those VAH patients who were asymptomatic, providing further evidence that an altered blood rheology in the vertebral artery could result in posterior cerebral ischemia [25].

\section{Discussion and Conclusion}

The prevalence of $\mathrm{VAH}$ is roughly estimated to range from 1.9 to $11.6 \%$, since neither consensus on a standardized measuring system nor on the cut-off diameter (range 2.0-3.0 $\mathrm{mm}$ ) for the definition of a VAH has been achieved to date. Thus, comparison of data from different research groups is difficult if not impossible $[12,26]$. Results from two separate ultrasound protocols indicate that right-sided VAH is twice as more common than left-sided VAH $[12,27]$. As for the degree of hypoplasia, severe unilateral hypoplasia was detected in $5.3 \%$ of the total study population and in $12.3 \%$ of the hypoplastic subgroup in MRI scans of patients with cervical pain [26]. VAH has also been related to basilar artery hypoplasia or stenosis, which further increases the risk of posterior circulation hypoperfusion [28].

Among imaging techniques, Doppler ultrasonography is a valuable, quick and noninvasive technique in the assessment of the vertebral arteries, even though the differential diagnosis between vertebral artery hypoplasia, aplasia, occlusion or dissection may be difficult [22]. Even conventional angiography or MRA may fail to distinguish reliably vertebral artery hypoplasia or aplasia from vascular occlusion, as they only demonstrate the intraluminal flow and cannot visualize the vascular walls [29]. Therefore, the difficulty for standard angiographic techniques to differentiate congenital variants from secondary thrombosis in the vertebral arteries leads primary to VAH underdiagnosis [30].
Caplan and Baker [31] were the first to notice that smaller of paired arteries are more likely to occlude compared with their larger counterparts, and raised the question if the congenital hypoplastic vessels are by nature more prone to occlusion. The aforementioned hypothesis was later stated in case reports from patients with posterior cerebral ischemia ipsilateral to a hypoplastic vertebral artery $[3,5-8]$ and further investigated in large cohort studies [15-19, 24].

Clearly the explanation cannot be size alone as many intracranial arteries are smaller than the hypoplastic or smaller arteries identified in these studies, and they are not predisposed to occlude. The answer must lie somehow in the physics of blood flow and sheer forces. There must be an interaction between blood pressure, blood constituents and the rheology and physics of blood flow at various arterial locations that explains the data [32]. A small-diameter artery appears to be more vulnerable to stenosis or occlusion, as its low flow velocity predisposes to prothrombotic or atherosclerotic processes in the presence of conventional vascular risk factors. Finally, a posterior circulation stroke may occur due to artery-to-artery embolism from the low-flowed stenotic VA [23]. Consistent literature data about the association of VAH with a specific pathogenetic mechanism do not exist. The hypothesis by Park and coworkers is in accordance with the clinical observation that proximal embolism (cardiac or arterial) is the leading cause of infarction in posterior circulation, especially in cases of superficial posterior cerebral artery infarction, with the source of embolism remaining undetermined in more than one third of the cases $[33,34]$. However, we may note that in our series of patients with VAH and medullary ischemia, in situ atherothrombosis in the territory of perforators could be the most likely mechanism [3].

Therefore, the posterior circulation is presumably more vulnerable to ischemia in patients with VAH, particularly in those with severe hypoplasia. Most of these individuals remain asymptomatic, but the stroke risk increases further when additional atherosclerotic factors coexist.

\section{Disclosure Statement}

The authors report no potential conflict of interest.
82

Eur Neurol 2013;70:78-83 DOI: $10.1159 / 000351786$
Katsanos/Kosmidou/Kyritsis/ Giannopoulos 


\section{References}

1 Cloud GC, Markus HS: Diagnosis and management of vertebral artery stenosis. Q J Med 2003;96:27-54.

2 Katsanos AH, Kosmidou M, Giannopoulos S: Vertebral artery hypoplasia in posterior circulation cerebral ischemia. Clin Neurol Neurosurg 2013;115:1194-1195.

3 Giannopoulos S, Markoula S, Kosmidou M, Pelidou SH, Kyritsis AP: Lateral medullary ischaemic events in young adults with hypoplastic vertebral artery. J Neurol Neurosurg Psychiatry 2007;78:987-989.

4 Giannopoulos S, Kosmidou M, Pelidou SH, Kyritsis AP: Vertebral artery hypoplasia. A predisposing factor for posterior circulation stroke? Neurology 2007;68:1956; author reply 1956-1957.

5 Orimo S, Goto K, Ozawa E, Murota T: Early recovery from locked-in syndrome due to brain infarction in a young patient with hypoplasia of bilateral vertebral arteries and a persistence of primitive trigeminal artery. Rinsho Shinkeigaku 1996;36:993-995.

6 Tai ML, Katiman E, Rahmat K, Tan CT: Acute bilateral medial medullary infarct with hypoplastic vertebral artery. Clin Neurol Neurosurg 2012;114:1365-1367.

7 Mestan MA: Posterior fossa ischemia and bilateral vertebral artery hypoplasia. J Manipulative Physiol Ther 1999;22:245-249.

8 Kawakami Y, Koizumi SY, Kuwabara K, et al: An 8-year-old boy with vertebral artery dissection with cerebellar ataxia featuring suspected vertebral artery hypoplasia. Brain Dev 2009;31:326-330.

9 Wang Y, Cai A, Liu L: Sonographic diagnosis of congenital variations of the extracranial vertebral artery and assessment of its circulation. J Ultrasound Med 2009;28:1481-1486.

10 Lovrencic-Huzjan A, Demarin V, Rundek T, Vukovic V: Role of vertebral artery hypoplasia in migraine. Cephalalgia 1998;18:684-686.

11 Simon M, Kafritsas D: Unilateral cerebellar hypoplasia. Clin Neuropathol 1992;11:71-73.

12 Chen YY, Chao AC, Hsu HY, Chung CP, Hu $\mathrm{HH}$ : Vertebral artery hypoplasia is associated with a decrease in net vertebral flow volume. Ultrasound Med Biol 2010;36:38-43.
13 Min JH, Lee YS: Transcranial Doppler ultrasonographic evaluation of vertebral artery hypoplasia and aplasia. J Neurol Sci 2007;260: 183-187.

14 Seidel E, Eicke BM, Tettenborn B, Krummenauer F: Reference values for vertebral artery flow volume by duplex sonography in young and elderly adults. Stroke 1999;30: 2692-2696.

15 Delcker A, Diener HC, Timmann D, Faustmann P: The role of vertebral and internal carotid artery disease in the pathogenesis of vertebrobasilar transient ischemic attacks. Eur Arch Psychiatry Clin Neurosci 1993;242:179183.

16 Tohgi H, Takahashi S, Chiba K, Hirata Y: Cerebellar infarction. Clinical and neuroimaging analysis in 293 patients. The Tohoku Cerebellar Infarction Study Group. Stroke 1993;24: 1697-1701.

17 Park JH, Kim JM, Roh JK: Hypoplastic vertebral artery: frequency and associations with ischaemic stroke territory. J Neurol Neurosurg Psychiatry 2007;78:954-958.

18 Perren F, Poglia D, Landis T, Sztajzel R: Vertebral artery hypoplasia: A predisposing factor for posterior circulation stroke? Neurology 2007;68:65-67.

19 Chuang YM, Huang YC, Hu HH, Yang CY: Toward a further elucidation: role of vertebral artery hypoplasia in acute ischemic stroke. Eur Neurol 2006;55:193-197.

$20 \mathrm{Hu}$ XY, Li ZX, Liu HQ, Zhang M, Wei ML, Fang S, Chen W, Pan H, Huang JX, Zhu YM, Liu JR: Relationship between vertebral artery hypoplasia and posterior circulation stroke in Chinese patients. Neuroradiology 2013;55: 291-295.

21 Bojinova V, Dimova P, Belopitova L: Clinical manifestations of cerebrovascular hypoplasias in childhood. J Child Neurol 2000;15: 166-171.

22 Buckenham TM, Wright IA: Ultrasound of the extracranial vertebral artery. Br J Radiol 2004;77:15-20.

23 Park JH, Roh JK, Kwon HM: Ischemic stroke patterns and hemodynamic features in patients with small vertebrobasilar artery. J Neurol Sci 2009;287:227-235.
24 Lochner P, Golaszewski S, Caleri F, et al: Posterior circulation ischemia in patients with fetaltype circle of Willis and hypoplastic vertebrobasilar system. Neurol Sci 2011;32:1143-1146.

25 Oder B, Oder W, Lang W, Marschnigg E, Deecke L: Hypoplasia, stenosis and other alterations of the vertebral artery. Does impaired blood rheology manifest a hidden disease? Acta Neurol Scand 1998;97:398-403.

26 Peterson C, Phillips L, Linden A, Hsu W: Vertebral artery hypoplasia. Prevalence and reliability of identifying and grading its severity on magnetic resonance imaging scans. J Manipulative Physiol Ther 2010;33:207-211.

27 Jeng JS, Yip PK: Evaluation of vertebral artery hypoplasia and asymmetry by color-coded duplex ultrasonography. Ultrasound Med Biol 2004;30:605-609.

28 Chaturvedi S, Lukovits TG, Chen W, Gorelick PB: Ischemia in the territory of a hypoplastic vertebrobasilar system. Neurology 1999;52: 980-983.

29 Takeuchi S, Wada K, Nagatani K, Nawashiro $\mathrm{H}$ : Differentiation between vertebral artery hypoplasia and occlusion. Intern Med 2012; 51:345.

30 Freund W, Kassubek J, Aschoff AJ, Huber R: Mri-based separation of congenital and acquired vertebrobasilar artery anomalies in ischemic stroke of the posterior circulation. Stroke 2008;39:2382-2384.

31 Caplan LR, Baker R: Extracranial occlusive vascular disease: does size matter? Stroke 1980;11:63-66.

32 Caplan LR: Arterial occlusions: does size matter? J Neurol Neurosurg Psychiatry 2007;78: 916.

33 Brandt T, Steinke W, Thie A, Pessin MS, Caplan LR: Posterior cerebral artery territory infarcts: clinical features, infarct topography, causes and outcome. Multicenter results and a review of the literature. Cerebrovasc Dis 2000;10:170-182.

34 Cals N, Devuyst G, Afsar N, Karapanayiotides T, Bogousslavsky J: Pure superficial posterior cerebral artery territory infarction in The Lausanne Stroke Registry. J Neurol 2002;249: 855-861. 